\title{
The German version of Early Childhood Oral Health Impact Scale (ECOHIS-G): translation, reliability, and validity
}

\author{
Katrin Bekes ${ }^{1}$ (D) $\cdot$ Maisa Omara ${ }^{2} \cdot$ Sabrina Safar $^{1} \cdot$ Tanja Stamm $^{2}$ \\ Received: 12 December 2018 / Accepted: 3 April 2019 / Published online: 16 April 2019 \\ (C) The Author(s) 2019
}

\begin{abstract}
Objectives The aims of this study were to develop a German version of the Early Childhood Oral Health Impact Scale (ECOHIS), a measure of oral health-related quality of life in pre-school-age children, and to assess the instrument's reliability and validity.

Methods The original English version of the ECOHIS questionnaire was translated into German (ECOHIS-G) by a forwardbackward translation method. A total of 336 children aged 0 to 5 years and their caregivers who were recruited at the Department of Paediatric Dentistry in Vienna, Austria, participated in this study. The caregivers completed the ECOHIS-G and their children were clinically examined for the presence of dental caries and plaque accumulation. Reliability of ECOHIS was investigated in a subsample of 59 subjects after 3 weeks.

Results Questionnaire summary score test-retest reliability was 0.81 (intraclass correlation coefficient, $95 \%$ confidence interval (CI) ranging from 0.68 to 0.89 ) and internal consistency was 0.83 (Cronbach's alpha, lower limit of the 95\% CI 0.80 ). Validity of the ECOHIS-G questionnaire was supported by correlation coefficients with global ratings of oral health of -0.42 (95\% CI 0.45 to -0.35$)$ and overall well-being of $-0.29(95 \% \mathrm{CI}-0.34$ to -0.22$)$ which met the expectations. Mean ECOHIS-G scores were statistically significantly higher in children with caries compared with caries-free children.

Conclusions The German version of the ECHOHIS was found to be reliable and valid in children aged 0 to 5 years.

Clinical relevance These findings enable assessments of oral health-related quality of life in German-speaking pre-school children.
\end{abstract}

Keywords Oral health-related quality of life (OHRQoL) $\cdot$ Early Childhood Oral Health Impact Scale (ECOHIS) $\cdot$ Psychometric properties $\cdot$ Reliability $\cdot$ Validity

\section{Introduction}

Nowadays, the concept of oral health-related quality of life (OHRQoL) has become an important measure to assess oral

Electronic supplementary material The online version of this article (https://doi.org/10.1007/s00784-019-02893-1) contains supplementary material, which is available to authorized users.

Katrin Bekes

katrin.bekes@meduniwien.ac.at

1 University Clinic of Dentistry, Department of Paediatric Dentistry, Medical University Vienna, Sensengasse 2a, 1090 Vienna, Austria

2 Center for Medical Statistics, Informatics, and Intelligent Systems, Section for Outcomes Research, Medical University of Vienna, Spitalgasse 23, 1090 Vienna, Austria health status in children and in adults [1] as clinical indicators alone do not reveal the full impact of oral conditions on the psychosocial well-being of a patient [2]. However, compared with a large number of instruments for adults [3], only a few OHRQoL instruments are available for children. Especially in young children, a special approach to measure OHRQoL is needed because their cognitive functions are not fully developed for filling out a questionnaire themselves. A common approach in this age is to ask their parents and potential other adult caregivers to complete the questionnaire. One of the most frequently used instrument to assess OHRQoL in preschool children is the Early Childhood Oral Health Impact Scale (ECOHIS), a questionnaire designed for adult caregivers, which was originally developed in the USA [4]. Different language versions have already been evaluated [5-10]; however, a German version did not exist to date. The ECOHIS showed good psychometric characteristics in 
terms of reliability and validity, making the questionnaire a promising tool for international collaboration, cross-border care, and linking of datasets for research. The Medical Outcomes Trust has made the existence of such instrument versions a criterion for the assessment and review of healthrelated quality-of-life instruments [11].

The aims of this study were therefore to develop a German version of the Early Childhood Oral Health Impact Scale and to assess the instrument's reliability and validity in $0-5$-yearold Austrian children.

\section{Materials and methods}

\section{Development of the German version of the ECOHIS}

The ECOHIS consists of 13 questions and is divided into two main parts, namely, the child impact section (9 items) and the family impact section (4 items). The child impact section contains four subscales: symptom (one item), child-related function (four items), psychology (two items), and self-image/social interaction (two items). The family impact section comprises two subscales: parental distress (two items) and familyrelated function (two items). Questions ask about the frequency of events in the child's entire life. Responses are made on an ordinal scale $(0=$ never, $1=$ hardly ever, $2=$ occasionally, 3 $=$ often, $4=$ very often). An additional answer option "do not know" exists in every item which was treated in the analysis like a missing answer. Summing the response codes for the questionnaire items generates domain scores/sub-scales and an overall ECOHIS score. Up to two missing responses on the child section or one missing on the family section, a score for the missing items could be imputed as an average of the remaining items for that section. Due to the psychometric nature of our analysis, e.g., assessing the reliability by comparing between answers of two time points, we decided not to impute missing items. The instrument's summary score ranges from 0 to 52; the scores for the child and family sections have a possible range from 0 to 36 and from 0 to 16 , respectively. A summary score of 0 indicates the absence of any problems, and higher ECOHIS scores represent worse OHRQoL. In addition to the 13 items, the ECOHIS includes two questions asking the caregiver for a global rating of the child's oral health and overall well-being. These global ratings have five response options (excellent, very good, good, moderate, poor).

Following accepted standards for the cross-cultural adaptation of health-related quality-of-life questionnaires [12], the original 13 English-language items were translated into German. Two independent native German speakers with extensive knowledge of English language and experience in translating health-related questionnaires carried out two independent translations. Both translations were merged into one version. This version was back-translated into English by two native English speakers. Finally, these two versions were synthesized and the final questionnaire (ECOHIS-G) was compared with the original English-language instrument. A committee consisting of two forward translators, two back-translators, and a methodologist supervised the entire translation process.

\section{Subjects and setting}

Children and their primary caregivers for this study were recruited from the Department of Paediatric Dentistry at the Medical University of Vienna, Vienna, Austria. To be included in the study, the children had to be aged 5 or younger, had no underlying serious medical conditions, did not take longterm medication, and were accompanied by a Germanspeaking primary caregiver. Primary caregivers of the children were invited to complete the ECOHIS-G. Caregivers whose children attended the clinic for a second time within 3 weeks for prophylaxis were invited to complete the ECOHIS-G a second time.

The children were clinically examined by one dentist who was trained and calibrated in accordance with the WHO Basic Methods criteria for visual assessment of dental caries [13]. The DMFT (sum of decayed, missing, and filled teeth in the permanent dentition) index and its components were used to assess caries status. Dental plaque accumulation was assessed using the simplified additive index for plaque accumulation described by Ambjørnsen et al. (score " 0 " = no visible plaque, score "1" = visible plaque (per participant/child) [14].

Approval for this study was obtained from the ethics committee of the local University Review Board (Medical University of Vienna; \#2015-1822). Written statements of consent were read and signed by the primary caregiver prior to their participation in the study.

\section{Reliability assessment}

The impact sections and the instrument's whole summary scores' internal consistency and temporal stability were analyzed. To determine internal consistency, Cronbach's alpha was calculated [15]. According to guidelines [16], Cronbach's alpha values of $0.70-0.80$ were considered "satisfactory" for a reliable comparison between groups. We compared Cronbach's alpha to the person separation index (PSI) which was calculated based on a Rasch model. PSI refers to the reproducibility of a relative measure location. A PSI value of $\geq 0.7$ indicates that the instrument is able to sufficiently distinguish between people with higher and lower OHRQoL [17]. In case of a skewed distribution of the total sum scores, e.g., when floor or ceiling effects appear, differences in Cronbach's alpha and PSI might occur. 
To determine the temporal stability of scores, we assessed test-retest reliability in a convenience sample of caregivers recruited at the Department of Paediatric Dentistry in Vienna. The interval between the first test interview and the retest interview was 3 weeks. The test-retest reliability was assessed by calculating intraclass correlation coefficients (ICC). We based the calculation of the ICCs on a two-way mixed-effect model because the two consecutive measurements (test-retest setting) were not randomized samples and assumed that there should be absolute agreement between the measurements of the two consecutive time points [18, 19]. According to guidelines [20], reliability coefficients $>0.75$ are considered excellent. For sample size calculation, we estimated that 51 individuals were required for detecting an ICC of 0.8 with a desired confidence interval width of 0.2 using the ICC package in R (www.r-project.org). We further estimated $15 \%$ drop outs and therefore aimed to include 59 participants for the test-retest analysis.

\section{Validity assessment}

To ensure that the instrument measured what it is supposed to measure, score validation was performed. We followed the approach taken by the developers of the original ECOHIS and those of other language versions to be compatible with previous validation efforts. Construct validity was determined by computing Spearman rank correlations of the caregiver's reported scores for the total scale and each domain with the global rating of oral health (excellent, very good, good, moderate, or poor) and reported global rating of overall well-being (excellent, very good, good, moderate, or poor). We used Spearman rank correlations because of non-normally distributed ECOHIS-G sum scores and the ordinally scaled global ratings of oral health and general well-being. It was assumed that children whose caregivers/parents rated their overall health and oral health status as poor would have higher ECOHIS-G scores. According to previous studies [4, 5, 7], we expected "weak" to "moderate" (0.10-0.29 and 0.30 0.49 , respectively, according to Cohen [21]) correlations between ECOHIS-G scores and the two global health questions, with a larger correlation with oral health than with general health.

In addition to our convergent validity analyses, we performed (known) group's validation where we determined to which degree ECOHIS-G scores are significantly different between groups of participants with different oral health status. We compared groups that differ regarding two major indicators of physical oral health: the caries index (dmft-Index; 0 versus $>0$ ) and the plaque accumulation index (absent versus present). We tested ECOHIS-G group differences using the Mann-Whitney-Wilcox test due to the non-normal distribution of the ECOHIS-G sum scores. Furthermore, we obtained point biserial correlation coefficients to determine the magnitude of the correlation between physical oral health indicators and ECOHIS scores. According to previous research, we expected differences in ECOHIS summary scores between subjects with more compared with less caries or plaque accumulation. The hypothesis was that caregivers of children with dental disease would score higher on the ECOHIS (indicating worse OHRQoL).

\section{Results}

\section{Development of the German ECOHIS-G}

All 13 items of ECOHIS were practicable to translate. Through focus group discussion, all items of the ECOHIS were considered comprehensible, clear, and relevant. The fifth item, "missed preschool, day care, or school" was adapted as "missed kindergarten, day care, or school" to provide conceptual equivalence of the item rather than a direct verbal equivalence as Austrian children attend a kindergarten or a day-care before entering school. Thus, we had to adopt these terms.

\section{Study population}

Of a total of 336 children initially selected, 95 (28\%) were excluded from the sample because their caregivers answered "do not know" to one or more items and/or the assessment of dental caries and plaque accumulation was missing. The final sample size included therefore 241 individuals, with a mean age of 3.7 years ( $\mathrm{SD}=1.2$, age range from 0 to 5 years). Of these, $128(53 \%)$ were girls. Fifty-nine patients completed the ECOHIS-G again after the test-retest time period of 3 weeks. The mean ECOHIS sum score of the 241 participants was 6.1 $( \pm 6.2$ ). In the test-retest group (59 participants), the mean ECOHIS sum score was $4.6( \pm 4.7)$ for the first assessment and $3.7( \pm 4.1)$ for the second assessment.

\section{Reliability}

In an examination of temporal stability in 59 patients, ECOHIS-G scores decreased slightly over the test-retest time period of 3 weeks (Table 1). For the subscales, the change in the child section was larger than the change observed for the family section. The ICC of the sum score was 0.81 (95\% CI ranging from 0.68 to 0.89 ) and 0.78 and 0.87 for the two subscales (Table 1) indicating "excellent" reliability $(>0.75)$ according to guidelines [20]. Internal consistency (Cronbach's alpha) for the summary score was 0.83 (with the lower limit of the $95 \%$ CI being 0.80) indicating a slightly higher than "satisfactory" reliability (Table 1) [16]. The PSI value was 0.80 and thus similar to Cronbach's alpha showing sufficient ability of the instrument to distinguish between people with higher and lower oral health impact. Average inter-item 
Table 1 ECOHIS score reliability in the study sample

\begin{tabular}{|c|c|c|c|c|c|}
\hline & \multirow[t]{2}{*}{ Number of items } & \multicolumn{2}{|c|}{ Internal consistency $(N=241)$} & \multicolumn{2}{|c|}{ Test-retest reliability $(N=59)$} \\
\hline & & $\begin{array}{l}\text { Cronbach's alpha } \\
\text { (lower limit of 95\% CI) }\end{array}$ & $\begin{array}{l}\text { Average inter-item } \\
\text { correlation }\end{array}$ & ICC $(95 \% \mathrm{CI})$ & $\begin{array}{l}\text { Mean ECOHIS score difference } \\
\text { examination time 1-time } 2(95 \% \mathrm{CI})\end{array}$ \\
\hline Total scale & 13 & $0.83(0.80)$ & 0.54 & $0.81(0.68-0.89)$ & $0.85(-0.08-1.79)$ \\
\hline \multicolumn{6}{|l|}{ Subscales } \\
\hline Child impact & 9 & $0.77(0.73)$ & 0.55 & $0.78(0.63-0.87)$ & $0.69(-0.01-1.39)$ \\
\hline Family impact & 4 & $0.76(0.72)$ & 0.65 & $0.87(0.78-0.92)$ & $0.17(-0.19-0.53)$ \\
\hline
\end{tabular}

correlation-another measure of scores' internal consistency_ranged between 0.54 and 0.65 .

\section{Validity}

ECOHIS scores correlated well with other measures of the same construct (convergent validity). The scores' correlations with global ratings of oral health and overall well-being were in the predicted magnitude (0.42 and 0.29, respectively) and were statistically significant (Table 2). As expected, the coefficient was higher for the rating of oral health than for the rating of overall well-being.

When children were grouped according to health indicators as caries and plaque accumulation, differences in ECOHIS scores were detected. Results of the assessment of discriminant validity indicated that, overall, children with either 0 or $\geq$ 0 decayed and/or treated teeth/surfaces had higher ECOHIS scores on both sections of the ECOHIS than those who were free of dental disease. As predicted, differences in children with and without plaque accumulation were also present and statistically significant (Table 2).

\section{Discussion}

This study adapted the original English language ECOHIS version to the German language and investigated its psychometric properties in Austrian children aged 0-5 years. The

Table 2 ECOHIS score validity in Austrian preschool children: correlations with caregiver-reported oral and general health as well as caries and plaque accumulation

\begin{tabular}{|c|c|c|c|c|c|c|c|}
\hline & \multirow[t]{2}{*}{$N$} & \multicolumn{2}{|c|}{ Child impact section (9 items) } & \multicolumn{2}{|c|}{ Family impact section (4 items) } & \multicolumn{2}{|c|}{ Overall scale (13 items) } \\
\hline & & $\begin{array}{l}\bar{x} \\
(\mathrm{SD})\end{array}$ & $\begin{array}{l}r \\
(95 \% \mathrm{CI})\end{array}$ & $\begin{array}{l}\bar{x} \\
(\mathrm{SD})\end{array}$ & $\begin{array}{l}r \\
(95 \% \mathrm{CI})\end{array}$ & $\begin{array}{l}\bar{x} \\
(\mathrm{SD})\end{array}$ & $\begin{array}{l}r \\
(95 \% \mathrm{CI})\end{array}$ \\
\hline Global rating of oral health & & & $-0.32(-0.40$ to -0.23$) *$ & & $-0.46(-0.58 \text { to }-0.36)^{*}$ & & $-0.42(-0.45 \text { to }-0.35)^{*}$ \\
\hline Excellent & 45 & $2.8(3.2)$ & & $0.9(1.7)$ & & $3.7(4.2)$ & \\
\hline Very good & 87 & $2.4(3.1)$ & & $1.2(2.2)$ & & $3.7(4.3)$ & \\
\hline Good & 53 & $4.9(4.6)$ & & $2.9(2.8)$ & & $7.8(6.5)$ & \\
\hline Moderate/poor** & 56 & $5.9(4.6)$ & & $4.3(3.5)$ & & $10.2(7.0)$ & \\
\hline \multicolumn{3}{|c|}{ Global rating of overall well-being } & $-0.26(-0.35 \text { to }-0.18)^{*}$ & & $-0.24(-0.36$ to -0.08$) *$ & & $-0.29(-0.34$ to -0.22$) *$ \\
\hline Excellent & 92 & $2.3(3.0)$ & & $1.3(2.1)$ & & $3.6(4.2)$ & \\
\hline Very good & 107 & $4.9(4.5)$ & & $2.5(2.9)$ & & $7.4(6.6)$ & \\
\hline Good & 30 & $4.9(4.1)$ & & $3.6(3.7)$ & & $8.5(6.8)$ & \\
\hline Moderate/poor** & 12 & $3.9(4.7)$ & & $3.8(4.2)$ & & $7.6(8.0)$ & \\
\hline & & $\begin{array}{l}\bar{x} \\
(\mathrm{SD})\end{array}$ & Median (IQR) & $\begin{array}{l}\bar{x} \\
(\mathrm{SD})\end{array}$ & Median (IQR) & $\begin{array}{l}\bar{x} \\
(\mathrm{SD})\end{array}$ & Median (IQR) \\
\hline \multicolumn{8}{|l|}{$\mathrm{dmft}$} \\
\hline 0 & 113 & $2.5(3.1)$ & $1(0$ to 4$)$ & $0.8(1.4)$ & $0(0$ to 1$)$ & $3.3(3.9)$ & $2(0$ to 5$)$ \\
\hline$\geq 1$ & 128 & $5.0(4.5)$ & $4(1-8) * ; \infty$ & $3.6(3.3)$ & $3(1-6)^{*} ; \circ$ & $8.6(6.7)$ & $7(3-13)^{*} ; \circ$ \\
\hline \multicolumn{8}{|l|}{ Plaque } \\
\hline absent & 144 & $3.3(4.0)$ & $2(0-5)$ & $1.8(2.9)$ & $0(0-2)$ & $5.1(6.1)$ & $3(1-7)$ \\
\hline present & 97 & $4.7(4.2)$ & $4(1-7)^{*} ; \circ$ & $2.9(2.8)$ & $2(0-5)^{*} ;{ }^{\circ}$ & $7.6(6.0)$ & $6(3-11)^{*} ;{ }^{\circ}$ \\
\hline
\end{tabular}

**Categories moderate and poor were combined because very few caregivers rated their children's oral health as "poor"; * $p<0.001 ;{ }^{\circ}$ Mann-WhitneyWilcox test to take into account the right skewed, non-normally distributed data 
translation and cross-cultural adaptation were carefully conducted following the four-step procedure recommended by Beaton et al. [12]. This process resulted in a back-translated version (ECOHIS-G) that was very similar to the original. The psychometric evaluation of the German version of the ECOHIS suggested good construct validity, discriminant validity, and internal consistency as well as excellent test-retest reliability.

Our results obtained from the reliability assessment were similar to findings from previous research. As quality-of-life assessment instruments should be reproducible over time [11], the analysis of test-retest reliability suggests the adequate stability of the instrument. For the evaluation, the time interval between the administrations of the questionnaires was restricted to 3 weeks in the current study. The test-retest reliability sample showed stability in responses to the ECOHIS-G. The ICC for the total scale was 0.81 and ranged from 0.78 to 0.87 for the sections, indicating excellent reproducibility according to guidelines, which was in agreement with previous studies that used a time interval of 2-3 weeks $[6,8,9]$. Moreover, it was higher than the values observed by Lee et al. in China [22]. In relation to internal consistency, Cronbach's alpha was satisfactory $(0.83,0.77$, and 0.76 for the ECOHIS total score and the child and family sections, respectively) indicating adequate reliability, as a Cronbach's alpha of 0.5 or above is considered acceptable $[15,20]$. Our internal consistency results were similar to findings in the French version $(\alpha=0.82$ for the summary score, 0.79 for both domain scores) [5] and the Brazilian version $(0.80$ and 0.76 for the child and family section, respectively) [7]. Compared with the original version presented by Pahel et al. [4] (0.91 and 0.95 for the child and family section, respectively), Cronbach's alpha was lower.

Construct validity of the German ECOHIS was as expected. Regarding convergent validity, the correlations between the global ratings of oral and overall well-being and the summary score were of moderate $(0.46)$ and weak (0.24) magnitude, respectively, statistically significant, and followed predicted patterns. Our findings were in agreement with the majority of previous ECOHIS validation studies that mainly investigated the correlation between oral health rating and ECOHIS scores, reporting that caregivers who thought their children had worse oral health were more likely to give their children higher ECOHIS scores. These investigations examining the English [4], French [5], and Turkish [8] versions also found moderate correlations, supporting suggestions that parents can provide valid reports for their children's OHRQOL when these conditions are observable. Furthermore, in the study examining the French version, also weak, but significant correlations were seen between global rating overall well-being and the summary score [5].
Discriminant validity was determined by comparing ECOHIS scores between children with and without caries experience and plaque accumulation being absent or present, respectively. We hypothesized that children with caries would have higher OHRQoL impacts. The results for our study indicated that Austrian children with caries did indeed have higher scores. Variations in the mean ECOHIS and the two subscale scores (child impact and family impact sections $)(p<0.001)$ were apparent. We found higher ECOHIS scores on both sections and the total scale among those with at least one carious lesion compared with those children who were caries free, indicating worse OHRQOL. This is consistent with two Brazilian studies [7, 23], one Indian study [24], and one Turkish study [8] which reported that children with dental caries experience obtained higher ECOHIS scores than those without. Also in agreement with our findings, Lee et al. [4] found a significant difference in both sections by testing the Chinese version. When we investigated the relationship of ECOHIS scores to plaque indicators, the findings pointed into a similar direction. Children with plaque being present were rated with significantly higher ECOHIS sum scores than those with plaque being absent.

\section{Conclusion}

The German version of the ECOHIS questionnaire is a valid and reliable instrument to measure OHRQoL of 0 5 -year-old children. The use of this instrument can help describe the impact of dental diseases (e.g., early childhood caries) and their treatment on pre-school children and their caregivers. Furthermore, it provides the opportunity to compare similarities and differences in oral health impacts among this age group in different countries.

Acknowledgements Open access funding provided by Medical University of Vienna. The authors are grateful to Mag. Iris Steinmetz (Medical University of Vienna) for her help with data management.

\section{Compliance with ethical standards}

Conflict of interest The authors declare that they have no conflict of interest.

Ethical approval All procedures performed in studies involving human participants were in accordance with the ethical standards of the institutional and/or national research committee and with the 1964 Helsinki declaration and its later amendments or comparable ethical standards.

This article does not contain any studies with animals performed by any of the authors.

Informed consent Informed consent was obtained from all individual participants included in the study. 
Open Access This article is distributed under the terms of the Creative Commons Attribution 4.0 International License (http:// creativecommons.org/licenses/by/4.0/), which permits unrestricted use, distribution, and reproduction in any medium, provided you give appropriate credit to the original author(s) and the source, provide a link to the Creative Commons license, and indicate if changes were made.

\section{References}

1. Bekes K, John MT, Zyriax R, Schaller HG, Hirsch C (2012) The German version of the Child Perceptions Questionnaire (CPQ-G1114): translation process, reliability, and validity in the general population. Clin Oral Investig 16:165-171. https://doi.org/10.1007/ s00784-010-0496-5

2. McGrath C, Broder H, Wilson-Genderson M (2004) Assessing the impact of oral health on the life quality of children: implications for research and practice. Community Dent Oral Epidemiol 32:81-85. https://doi.org/10.1111/j.1600-0528.2004.00149.x

3. Locker D, Allen F (2007) What do measures of 'oral health-related quality of life' measure? Community Dent Oral Epidemiol 35:401411. https://doi.org/10.1111/j.1600-0528.2007.00418.x

4. Pahel BT, Rozier RG, Slade GD (2007) Parental perceptions of children's oral health: the Early Childhood Oral Health Impact Scale (ECOHIS). Health Qual Life Outcomes 5:6. https://doi.org/ 10.1186/1477-7525-5-6

5. Li S, Veronneau J, Allison PJ (2008) Validation of a French language version of the Early Childhood Oral Health Impact Scale (ECOHIS). Health Qual Life Outcomes 6:9. https://doi.org/10. 1186/1477-7525-6-9

6. Farsi NJ, El-Housseiny AA, Farsi DJ, Farsi NM (2017) Validation of the Arabic version of the Early Childhood Oral Health Impact Scale (ECOHIS). BMC Oral Health 17(60):60. https://doi.org/10. 1186/s12903-017-0353-x

7. Scarpelli AC, Oliveira BH, Tesch FC, Leao AT, Pordeus IA, Paiva SM (2011) Psychometric properties of the Brazilian version of the Early Childhood Oral Health Impact Scale (B-ECOHIS). BMC Oral Health 11:19. https://doi.org/10.1186/1472-6831-11-19

8. Peker K, Uysal O, Bermek G (2011) Cross-cultural adaptation and preliminary validation of the Turkish version of the Early Childhood Oral Health Impact Scale among 5-6-year-old children. Health Qual Life Outcomes 9:118. https://doi.org/10.1186/14777525-9-118

9. Jabarifar SE, Golkari A, Ijadi MH, Jafarzadeh M, Khadem P (2010) Validation of a Farsi version of the early childhood oral health impact scale (F-ECOHIS). BMC Oral Health 10:4. https://doi.org/ 10.1186/1472-6831-10-4

10. Bordoni N, Ciaravino O, Zambrano O, Villena R, Beltran-Aguilar E, Squassi A (2012) Early Childhood Oral Health Impact Scale
(ECOHIS). Translation and validation in Spanish language. Acta Odontol Latinoam 25:270-278

11. Scientific Advisory Committee of the Medical Outcomes Trust (2002) Assessing health status and quality-of-life instruments: attributes and review criteria. Qual Life Res 11:193-205

12. Beaton RD, Johnson LC (2002) Instrument development and evaluation of domestic preparedness training for first responders. Prehosp Disaster Med 17:119-125

13. World Health Organization (1997) Oral health surveys. Basic methods. World Health Organization, Geneva

14. Ambjornsen E, Valderhaug J, Norheim PW, Floystrand F (1982) Assessment of an additive index for plaque accumulation on complete maxillary dentures. Acta Odontol Scand 40:203-208

15. Cronbach LJ (1951) Coefficient alpha and the internal structure of tests. Psychometrika 16:297-334

16. Bland JM, Altman DG (1986) Statistical methods for assessing agreement between two methods of clinical measurement. Lancet 1:307-310

17. Tennant A, Conaghan PG (2007) The Rasch measurement model in rheumatology: what is it and why use it? When should it be applied, and what should one look for in a Rasch paper? Arthritis Care Res 57:1358-1362

18. Shrout PE, Fleiss JL (1979) Intraclass correlations: uses in assessing rater reliability. Psychol Bull 86:420-428

19. Koo TK, Li MY (2016) A guideline of selecting and reporting intraclass correlation coefficients for reliability research. J Chiropract Med 15:155-163

20. Nunnally JC, Bernstein IR (1994) Psychometric theory. McGrawHill, New York

21. Cohen J (1988) Statistical power analysis for the behavioral sciences. Lawrence Erlbaum, Hillsdale, NJ

22. Lee GH, McGrath C, Yiu CK, King NM (2009) Translation and validation of a Chinese language version of the early childhood Oral health impact scale (ECOHIS). Int J Paediatr Dent 19:399 405. https://doi.org/10.1111/j.1365-263X.2009.01000.x

23. Martins-Junior PA, Ramos-Jorge J, Paiva SM, Marques LS, Ramos-Jorge ML (2012) Validations of the Brazilian version of the Early Childhood Oral Health Impact Scale (ECOHIS). Cad Saude Publica 28:367-374

24. Bhat SG, Sivaram R (2015) Psychometric properties of the Malayalam version of ECOHIS. J Indian Soc Pedod Prev Dent 33:234-238. https://doi.org/10.4103/0970-4388.160398

Publisher's note Springer Nature remains neutral with regard to jurisdictional claims in published maps and institutional affiliations. 